\title{
Reattempt Percutaneous Coronary Intervention of Chronic Total Occlusions after Prior Failures: A Single-Center Analysis of Strategies and Outcomes
}

\author{
Mingqiang Fu (D), Shufu Chang $(\mathbb{D}$, Lei Ge, Dong Huang, Kang Yao, Feng Zhang, Qing Qin, \\ Jianying Ma $\mathbb{D}$, Juying Qian, and Junbo Ge $\mathbb{D}$
}

Department of Cardiology, Zhongshan Hospital, Fudan University, Shanghai Institute of Cardiovascular Diseases, Shanghai, China

Correspondence should be addressed to Jianying Ma; ma.jianying@zs-hospital.sh.cn and Junbo Ge; jbge@zs-hospital.sh.cn

Received 27 September 2020; Revised 24 March 2021; Accepted 9 April 2021; Published 20 April 2021

Academic Editor: Stefano Rigattieri

Copyright ( $\odot 2021$ Mingqiang Fu et al. This is an open access article distributed under the Creative Commons Attribution License, which permits unrestricted use, distribution, and reproduction in any medium, provided the original work is properly cited.

\begin{abstract}
Objective. The initial recanalization rate of coronary chronic total occlusions (CTOs) is $>85 \%$ when performed by experienced operators, but only $10 \%$ of prior failed CTO patients receive reattempted recanalization. This retrospective study analyzed the success rate and strategies used in reattempt percutaneous coronary intervention (PCI) of CTOs after prior failures. Methods. Overall, 206 patients with 212 CTOs were enrolled. All patients with prior recanalization failures received reattempt PCIs from January 2015 to March 2019 at Zhongshan Hospital, Fudan University. Data on clinical factors (age, sex, comorbidities, left ventricular ejection fraction, history of cigarette usage, and revascularization), angiographic characteristics of CTOs (target lesion, Japanese Chronic Total Occlusion (J-CTO) score, the morphology of CTO lesions, and collateral channel scale), strategies (procedural approach and use of devices), and major adverse events were obtained and analyzed. Results. The mean age of enrolled patients was $60.96 \pm 12.36$ years, with a male predominance of $90.3 \%$. Of the patients, $47.1 \%$ had a prior myocardial infarction and $70.4 \%$ underwent stent implantation previously, while the in-stent occlusion rate was $6.6 \%$. CTOs were primarily localized in the left anterior descending artery (43.9\%) and the right coronary artery (43.9\%). 80.7\% of lesions were classified as very difficult (J-CTO score $\geq 3$ ), and the overall success rate was $81.1 \%$. In multivariable regression analysis, J-CTO score, collateral channel scale, application of coronary multispiral computed tomography angiography, dual injection, intravascular ultrasound, active greeting technique, parallel wiring, and CTO morphology were predictors of recanalization success. There were no significant differences in rates of procedural complications between the final recanalization success and failure groups. Conclusions. Recanalization of complex CTOs is associated with high success rate and low complication rates when performed by high-volume CTO operators and after multiple reattempts.
\end{abstract}

\section{Introduction}

Chronic total occlusion (CTO) lesions are identified in $18 \%-$ $33 \%$ of all patients referred for coronary angiography $[1,2]$. Also, the presence of concurrent CTO is a strong predictor for both short-term and long-term mortality [3,4]. In the era of interventional therapy, the indications for CTO revascularization are similar to those for severe stenosis according to European Society of Cardiology guidelines [5]. Compared with optimal medical therapy alone, the combination of CTO revascularization with optimal medical therapy is associated with significant ischemia relief, left ventricular function improvement, and a better quality of life [6-8]. In recent years, the development of contemporary techniques and devices has substantially improved the initial success rate $>85 \%$ for CTO-PCI in unselected clinically indicated cases with $\approx 3 \%$ risk for major in-hospital complications when performed by highly experienced operators [9-11]. However, outcomes are less favorable at less-experienced centers with an initial success rate of around $60 \%[12,13]$; the procedural failed CTO patients either receive medical therapy still suffering symptomatic ischemia or are advised 
to undergo reattempt PCI or more traumatic coronary artery bypass grafting (CABG) surgery [14].

It is unclear whether reattempted PCI for CTO lesions is efficacious and safe by expert operators because the prior failure of percutaneous revascularization of CTO has been identified as an independent predictor for failure at subsequent attempts [15]. To our knowledge, there have been scattered reports focusing on strategies and outcomes for reattempted CTO-PCIs following previously failed procedures. In the present study, we sought to define outcomes and predictors of reattempted CTO-PCI success performed by highly skilled operators at our center.

\section{Methods}

2.1. Study Population and Data Collection. Between January 1, 2015, and March 31, 2019, a total of 206 consecutive patients with CTO lesions after prior PCI failures at local hospitals or our center with a history of multiple recanalization attempts were included. Patient data on baseline clinical characteristics, coronary angiographic information, procedural strategies, and complications were obtained.

2.2. Study Definitions and Evaluation of Strategies and Outcomes. Coronary chronic total occlusions (CTOs) are defined as $100 \%$ occlusions with Thrombolysis in Myocardial Infarction (TIMI) 0 flow with at least a 3-month duration $[2,16]$. Occlusion duration was estimated based on the first onset of ischemic symptoms, prior history of myocardial infarction (MI) in the target vessel territory, comparison with a prior angiogram, or as the presence of bridging collateral vessels.

Lesion calcification was classified as mild (spots), moderate ( $\leq 50 \%$ of the reference lesion diameter), or severe ( $>50 \%$ of the reference lesion diameter). Moderate proximal vessel tortuosity was defined as the presence of at least 2 bends $>70^{\circ}$ or 1 bend $>90^{\circ}$, and severe tortuosity was defined as 2 bends $>90^{\circ}$ or 1 bend $>120^{\circ}$ in the CTO vessel. Blunt or no stump was defined as the absence of tapering or a funnel shape at the proximal or distal cap.

Collaterals included septal, epicardial, ipsilateral, and saphenous vein graft. Angiographic assessment of a collateral connection (CC) was based on Werner's classification: $\mathrm{CC} 0$, no continuous connection between the donor and recipient artery; $\mathrm{CC} 1$, continuous, thread-like connection; and CC2, continuous small side branch-like size of the collateral throughout its course [17].

The J-CTO (Multicenter CTO Registry in Japan) score was determined by assigning 1 point to each of the following factors: blunt entry stump, calcification, bend $>45^{\circ}$, occlusion length $\geq 20 \mathrm{~mm}$, and previous failed attempt. The total number of points was added to stratify lesions into 4 difficulty groups: easy (J-CTO score of 0 ), intermediate (J-CTO score of 1 ), difficult (J-CTO score of 2 ), and very difficult (JCTO score $\geq 3$ ) [18].

Recanalization strategies of CTO include two paths (anterograde or retrograde) and two ways: through the true lumen or the subintimal space (with dissection and reentry to the true lumen). The hybrid strategy involved using both antegrade and retrograde approaches or switching from the originally selected approach to the other approach $[19,20]$. The operators determined which strategy to use.

Technical success was defined as the ability to cross an occluded segment and successfully open the artery (restoration of TIMI flow grade of 2 or 3 ) with residual stenosis of $<30 \%$. Procedural success was defined as the achievement of technical success without any in-hospital major adverse cardiac events (MACE) [19].

Procedural complications included coronary artery dissection, hematoma and/or perforation, and tamponade requiring either pericardiocentesis or surgery. Contrastinduced nephropathy (CIN) was defined as an absolute increase of $\geq 0.5 \mathrm{mg} / \mathrm{dL}$ or a relative increase of at least $25 \%$ in serum creatinine levels within 48 to $72 \mathrm{~h}$ of intravenous administration of an iodinated contrast agent in the absence of other identifiable causes [21]. In-hospital MACE included any of the following before discharge: death, MI (defined using the fourth universal definition of type $4 \mathrm{a}$ ), and recurrent symptoms requiring immediate repeat target vessel revascularization with $\mathrm{PCI}$ or CABG.

2.3. Statistical Analysis. Categorical variables are presented as numbers (percentages) and compared using Pearson chisquare or Fisher exact tests. Continuous variables are presented as mean \pm standard deviation if normally distributed or median with an interquartile range if nonnormally distributed and compared using Student's $t$ or Mann-Whitney $\mathrm{U}$ test, as appropriate. All indices with a $p$ value $<0.1$ in the univariate analysis were included in a multivariable logistic regression analysis to test reattempted CTO-PCI success with baseline clinical and angiographic characteristics as well as strategies. All data were analyzed using SPSS 20.0 (IBM SPSS Inc., Armonk, NY). A two-sided $p$ value of 0.05 was considered statistically significant.

\section{Results}

3.1. Clinical Characteristics. A total of 206 patients were enrolled in the study. Nearly $90 \%$ of the patients for reattempts (178 out of 206 patients) were referred from other centers or other operators. Reattempts of CTOs were required to achieve recanalization in 166 patients, while recanalization could not be achieved in 40 patients even after multiple reattempts. The mean age of patients was $60.96 \pm 12.36$ years, with a male predominance of $90.3 \%$. Regarding comorbidities, $69.4 \%$ had hypertension, $33.5 \%$ had diabetes mellitus, 20.9\% had hyperlipidemia, 54.9\% were smokers, and $47.1 \%$ had prior MI. Notably, $70.4 \%$ of patients had previously received stent implantation, and 5.8\% had received CABG. There were no significant differences in the distribution of clinical characteristics between patients with final successful reattempts and final failed reattempts (Table 1 ).

3.2. Angiographic Characteristics. There were 212 initially failed CTOs in 206 patients, of which 172 CTOs in 166 patients were successfully recanalized after reattempts, 
TABle 1: Baseline clinical characteristics of the patients.

\begin{tabular}{|c|c|c|c|c|}
\hline Category & Overall $(n=206)$ & Final success $(n=166)$ & Final failure $(n=40)$ & $p$ value \\
\hline Age (years) & $60.96 \pm 12.36$ & $61.04 \pm 12.34$ & $60.62 \pm 12.60$ & 0.851 \\
\hline Male (\%) & $186(90.3)$ & $150(90.4)$ & $36(90.0)$ & 0.945 \\
\hline HTN (\%) & $143(69.4)$ & $111(66.9)$ & $32(80.0)$ & 0.106 \\
\hline $\mathrm{DM}(\%)$ & $69(33.5)$ & $58(34.9)$ & $11(27.5)$ & 0.371 \\
\hline HL (\%) & $43(20.9)$ & $35(21.1)$ & $8(20.0)$ & 0.880 \\
\hline Smokers (\%) & $113(54.9)$ & $93(56.0)$ & $20(50.0)$ & 0.492 \\
\hline LVEF (\%) & $56.37 \pm 9.90$ & $55.99 \pm 9.71$ & $57.97 \pm 10.61$ & 0.255 \\
\hline$\leq 40(\%)$ & $17(8.3)$ & $13(7.8)$ & $4(10.0)$ & 0.655 \\
\hline$>40(\%)$ & $189(91.7)$ & $153(92.2)$ & $36(90.0)$ & 0.655 \\
\hline eGFR $\left(\mathrm{mL} / \mathrm{min} / 1.73 \mathrm{~m}^{2}\right)$ & $82.71 \pm 22.12$ & $83.25 \pm 21.49$ & $80.45 \pm 24.73$ & 0.473 \\
\hline$\leq 60(\%)$ & $36(17.5)$ & $28(16.9)$ & $8(20.0)$ & 0.640 \\
\hline$>60(\%)$ & $170(82.5)$ & $138(83.1)$ & $32(80.0)$ & 0.640 \\
\hline Prior MI (\%) & $97(47.1)$ & $74(44.6)$ & $23(57.5)$ & 0.142 \\
\hline Prior PCI (\%) & $145(70.4)$ & $120(72.3)$ & $25(62.5)$ & 0.223 \\
\hline Prior CABG (\%) & $12(5.8)$ & $12(7.2)$ & $0(0.0)$ & 0.169 \\
\hline
\end{tabular}

Values are mean \pm SD or percentages. $p$ value stands for comparison between final successful and final failed patients' clinical characteristics. HTN, hypertension; DM, diabetes mellitus; HL, hyperlipidemia (low-density lipoprotein cholesterol $\geq 3.4 \mathrm{mmol} / \mathrm{L}$ ); LVEF, left ventricular ejection fraction; eGFR, estimated glomerular filtration rate; MI, myocardial infarction; PCI, percutaneous coronary intervention; CABG, coronary artery bypass grafting.

whereas recanalization of 40 CTOs in 40 patients finally failed. Typically, target CTO lesions were primarily located in the left anterior descending coronary artery (43.9\%) or right coronary artery (43.9\%), and $6.6 \%$ were in-stent occlusions. Of the CTOs, $54.2 \%$ scaled CC grade 1 and $43.9 \%$ scaled CC grade 2, and more than two-thirds were multiple coronary vessel disease. Among the included CTOs, $80.7 \%$ were technically very difficult (J-CTO score $\geq 3$, prior failure as part of the score derivation). By detailed evaluation of each characteristic of J-CTO score, $75.5 \%$ were occlusion lesions $>20 \mathrm{~mm}, 27.8 \%$ were tortuous, $47.2 \%$ were blunt proximal cap, $24.1 \%$ were blunt distal cap, and $9.4 \%$ were severely calcified.

Compared with final successful procedures, final failed lesions had considerably higher J-CTO score ( $4.00 \pm 0.72$ vs. $3.33 \pm 0.87, p<0.001$ ), less-useful CCs (CC grade $0: 7.5 \%$ vs. $0.6 \%, p=0.004$; CC grade $2: 27.5 \%$ vs. $47.7 \%, p=0.021$ ), and higher triple vessel disease rate $(67.5 \%$ vs. $23.5 \%, p<0.001)$, which indicated the final failed lesions had more complicated morphology (Table 2).

3.3. Strategies between Successful and Failed Cases. Of all reattempted CTO procedures, second-attempt recanalization was achieved in 165 lesions, third-attempt recanalization was acquired in 6 lesions, and fourth-attempt recanalization was successful in 1 lesion, and the overall success rate was $81.1 \%$ (172/212 lesions; Figure 1).

At initial attempts, antegrade wiring was required in $79.2 \%(168 / 212)$ of patients, with a dual injection rate of $18.4 \%$ (39/212). When reattempts were implemented, antegrade wiring was required in $48.6 \%(103 / 212)$ of patients, with a dual injection rate of $67.9 \%(144 / 212)$. The most used collateral in retrograde wiring was the septal connection (42.0\%). 10.8\% (23/212) of reattempted cases involved tip injection, $38.7 \%(82 / 212)$ cases required intravascular ultrasound (IVUS) to facilitate CTO wiring, and $16.0 \%(34 / 212)$ cases required reverse-controlled antegrade or retrograde subintimal tracking (R-CART).
CrossBoss and/or Stringray devices (Boston Scientific Corporation) were used in 13 cases (6.1\%), and active greeting technique (AGT) was used in 26 cases (12.3\%).

The intervals for a second recanalization attempt between finally successful and failed cases were comparable $(4.08 \pm 5.26$ months vs. $3.85 \pm 5.34$ months, $p=0.799)$. Compared with final failed cases, final successful cases involved greater apply of coronary multispiral computed tomography angiography (CCTA, $27.1 \%$ vs. $7.5 \%, p<0.001$ ) and dual injection $(73.8 \%$ vs. $42.5 \%, p=0.003)$ to acquire comprehensive anatomical information of target lesions. Meanwhile, implementation of IVUS, R-CART, and AGT was more common in final successful cases. There were no significant differences in comparison to the procedural approach, collaterals used, rotational atherectomy and anterograde dissection and reentry (ADR; use of CrossBoss and/or Stringray CTO reentry devices) maneuvers (Table 3).

3.4. Safety and Complications. As is presented in Table 4, reattempts were accompanied by greater contrast and X-ray doses than the initial attempts. Compared to finally recanalized lesions, final failed cases required more contrast $(317.38 \pm 184.42 \mathrm{ml}$ vs. $297.38 \pm 148.96 \mathrm{ml}, p<0.05)$ and increased dose of X-ray $(2491.28 \pm 1178.14 \mathrm{mGy}$ vs. $2319.11 \pm 1397.31 \mathrm{mGy}, p<0.05)$ and were time consuming $(48.82 \pm 33.66 \mathrm{~min}$. vs. $46.89 \pm 34.10 \mathrm{~min} ., \quad p<0.05)$.

$7.5 \%$ of patients in the final failed group experienced tamponade during the procedure, while other procedurerelated complications and MACE were comparable between final successful and failed cases. Notably, the contrast-induced nephropathy (CIN) rate was considerably higher in the final successful group patients than in the final failed group patients (Table 5).

3.5. Reasons for Initial Procedural Failure and Predictors of Success. The reasons for initial procedural failures are summarized in Figure 2; wire failure was the predominant 
TABLE 2: Baseline angiographic characteristics of CTO lesions.

\begin{tabular}{|c|c|c|c|c|}
\hline Category & Overall $(n=212)$ & Final success $(n=172)$ & Final failure $(n=40)$ & $p$ value \\
\hline \multicolumn{5}{|l|}{ CTO target vessels } \\
\hline LM (\%) & $1(0.4)$ & $1(0.6)$ & $0(0.0)$ & 0.629 \\
\hline $\mathrm{LAD}(\%)$ & $93(43.9)$ & $79(45.9)$ & $14(35.0)$ & 0.210 \\
\hline LCX (\%) & $25(11.8)$ & $20(11.6)$ & $5(12.5)$ & 0.878 \\
\hline RCA (\%) & $93(43.9)$ & $72(41.9)$ & $21(52.5)$ & 0.222 \\
\hline In-stent occlusion (\%) & $14(6.6)$ & $10(5.8)$ & $4(10.0)$ & 0.337 \\
\hline \multicolumn{5}{|l|}{ Collateral channels } \\
\hline CC $0(\%)$ & $4(1.9)$ & $1(0.6)$ & $3(7.5)$ & 0.004 \\
\hline CC $1(\%)$ & $115(54.2)$ & $89(51.7)$ & $26(65.0)$ & 0.130 \\
\hline CC $2(\%)$ & $93(43.9)$ & $82(47.7)$ & $11(27.5)$ & 0.021 \\
\hline J-CTO score & $3.45 \pm 0.88$ & $3.33 \pm 0.87$ & $4.00 \pm 0.72$ & $<0.001$ \\
\hline$<3(\%)$ & $41(19.3)$ & $33(19.2)$ & $8(20.0)$ & 0.907 \\
\hline$\geq 3(\%)$ & $171(80.7)$ & $139(80.8)$ & $32(80.0)$ & 0.907 \\
\hline \multicolumn{5}{|l|}{ Number of diseased vessels } \\
\hline Single VD & $44(21.4)$ & $41(24.7)$ & $3(7.5)$ & 0.022 \\
\hline Double VD & $84(40.8)$ & $75(45.2)$ & $9(22.5)$ & 0.014 \\
\hline Triple VD & $66(32.0)$ & $39(23.5)$ & $27(67.5)$ & $<0.001$ \\
\hline LM + multiple VD & $12(5.8)$ & $11(6.6)$ & $1(2.5)$ & 0.471 \\
\hline \multicolumn{5}{|l|}{ Morphology of the proximal cap } \\
\hline Blunt & $100(47.2)$ & $70(40.7)$ & $30(75.0)$ & $<0.001$ \\
\hline Side branch at the proximal cap & $153(72.2)$ & $118(68.6)$ & $35(87.5)$ & 0.016 \\
\hline \multicolumn{5}{|l|}{ Target lesion morphology } \\
\hline Tortuosity of the CTO lesion & $59(27.8)$ & $47(27.3)$ & $12(30.0)$ & 0.734 \\
\hline CTO length $\geq 20 \mathrm{~mm}$ & $160(75.5)$ & $122(70.9)$ & $38(95.0)$ & 0.001 \\
\hline \multicolumn{5}{|l|}{ Lesion calcification } \\
\hline Mild & $169(79.7)$ & $141(81.9)$ & $28(70.0)$ & 0.090 \\
\hline Moderate & $23(10.9)$ & $19(11.1)$ & $4(10.0)$ & 0.848 \\
\hline Severe & $20(9.4)$ & $12(7.0)$ & $8(20.0)$ & 0.025 \\
\hline \multicolumn{5}{|l|}{ Morphology of the distal cap } \\
\hline Blunt & $51(24.1)$ & $24(14.0)$ & $27(67.5)$ & $<0.001$ \\
\hline Side branch at the distal cap & $83(39.2)$ & $59(34.3)$ & $24(60.0)$ & 0.003 \\
\hline
\end{tabular}

Values are mean \pm SD or percentage. $p$ value stands for comparison between final successful and final failed angiographic characteristics of CTO lesions. LM, left main artery; LAD, left anterior descending coronary artery; LCX, left circumflex coronary artery; RCA, right coronary artery; CC, collateral connection; $\mathrm{VD}$, vessel disease.

reason to terminate the procedure $(84.5 \%)$, followed by a contrast limit (6.1\%). Rare reasons included radiation limit, operator or patient fatigue, and microcatheter failure.

To determine which indexes were mainly affecting procedural outcomes, we conducted correlation and logistic regression analyses. Multivariable regression analysis revealed that J-CTO score, CC scale, CCTA, dual injection, IVUS, AGT, parallel wiring, and CTO morphology were predictors of recanalization success. In contrast, sex, age, target lesion location, time of interval, procedural approach, ADR/RDR, rotational atherectomy, bridging collaterals, in-stent occlusion, CTO bend, comorbidities, smoke, LVEF, renal function, and previous histories of $\mathrm{MI}$ and revascularization appeared less important (Table 6). Correlation analysis revealed that higher J-CTO score, collaterals existed in proximal or distal lesions, lesion length $\geq 20 \mathrm{~mm}$, and severe lesion calcification were negatively correlated with procedural success. In contrast, higher CC scale, preoperation CCTA application, dual injection, IVUS-guided wiring, AGT, and tapered cap existed in both proximal and distal lesions were positively correlated with successful recanalization (supplementary table).

\section{Discussion}

The main findings of the present study were that prior failed CTO lesions were associated with higher complexity of morphology; however, $81.1 \%$ of CTOs could be recanalized safely and effectively by experienced operators at repeat attempts. There was a definite relationship between lesion complexity and the increasing need for multiple approaches and technologies during CTO-PCI to achieve success.

Multicenter CTO Registry in Japan proved that "retry" was an unsuccessful predictor for initial failed CTOs [15]; on the other hand, CTO patients were less likely to choose for re-PCI potentially due to low expectations of procedural success and concerns regarding complications; thus, merely $10 \%$ patients were referred for PCI other than optimal medical therapy or CABG [22]. A meta-analysis of 25 studies compared successful (71\%) with failed (29\%) CTO-PCIs in 28486 patients. During a mean follow-up of 3.11 years, successful CTO-PCI was associated with lower mortality, less residual angina, lower risk for stroke, and less need for subsequent CABG with comparison to failed procedures 


\begin{tabular}{|c|c|c|c|c|c|c|c|c|c|}
\hline \multicolumn{4}{|c|}{ Antegrade approach $(n=168$} & Retrograde approach $(n=4)$ & \multicolumn{5}{|c|}{ Hybrid approach $(n=40)$} \\
\hline 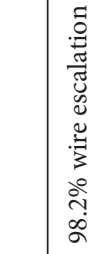 & 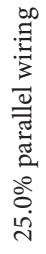 & 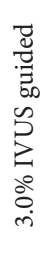 & 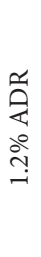 &  & 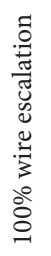 & 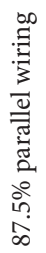 &  & 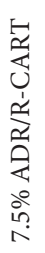 & 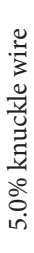 \\
\hline
\end{tabular}

\begin{tabular}{|c|c|c|c|c|c|c|c|c|c|c|c|}
\hline \multicolumn{5}{|c|}{ Antegrade approach $(n=104)$} & Retrograde approach $(n=20)$ & \multicolumn{6}{|c|}{ Hybrid approach $(n=88)$} \\
\hline 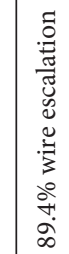 & 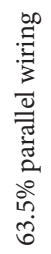 & 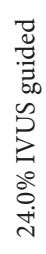 & $\begin{array}{l}\text { 告 } \\
\text { 구 } \\
\infty \\
\infty \\
\infty \\
\text { in }\end{array}$ & 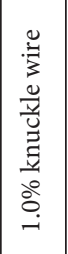 & 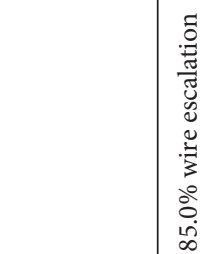 & 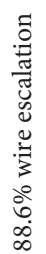 & 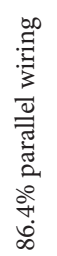 &  & 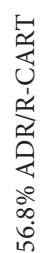 & 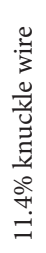 & $\begin{array}{l}\text { ப) } \\
\text { in } \\
\text { ì }\end{array}$ \\
\hline
\end{tabular}

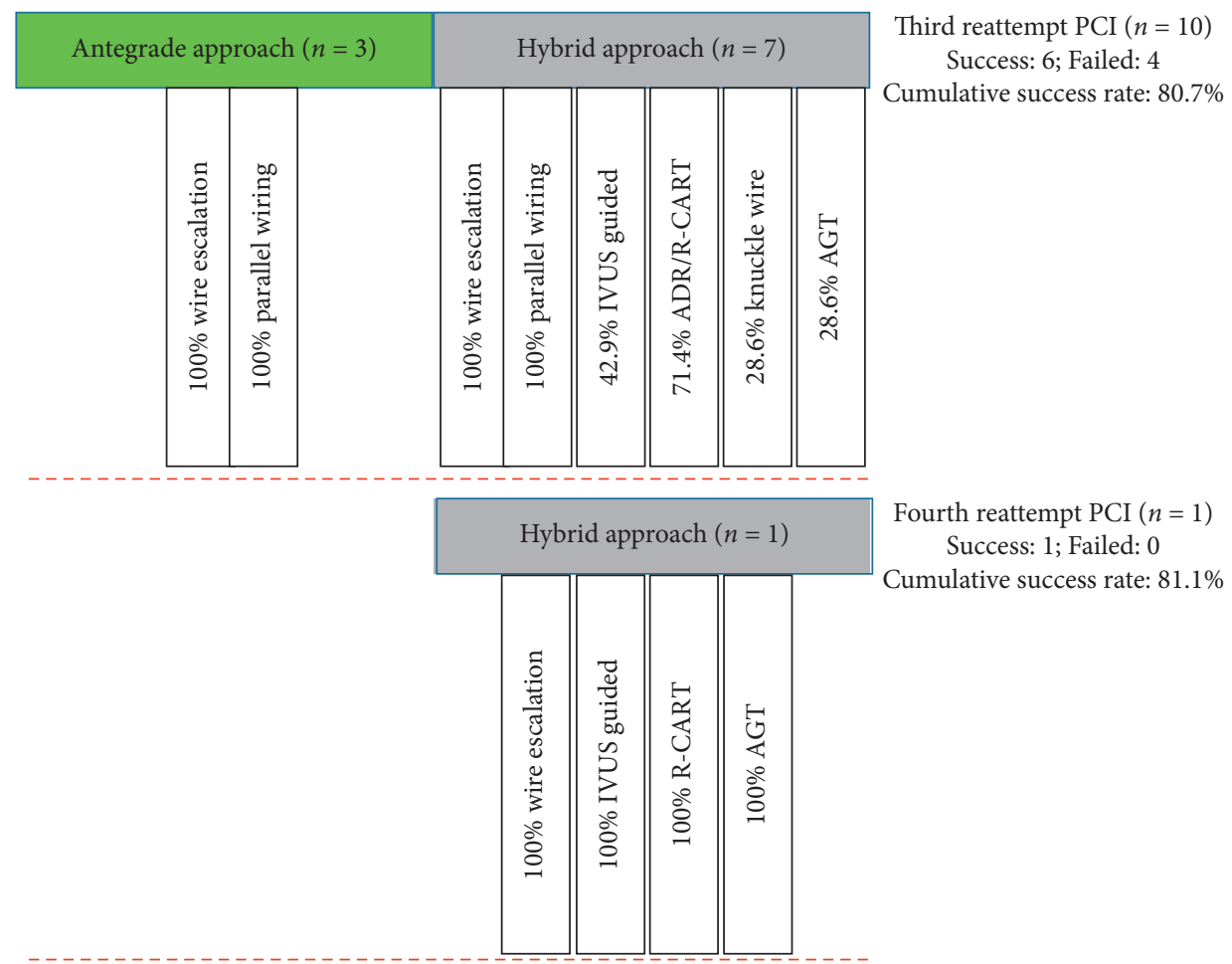

FIGURE 1: Distributions of strategies and crossing techniques. PCI, percutaneous coronary intervention; IVUS, intravascular ultrasound; ADR, antegrade dissection and reentry (CrossBoss and/or Stingray device); R-CART, reverse-controlled antegrade or retrograde subintimal tracking; and AGT, active greeting technique.

[23]. According to related guidelines and from our experience, successful opening of CTOs, even repeated attempts, would greatly benefit patients with fewer complications.

Generally, initial failure of recanalization for CTOs indicates that lesion morphologies are more unfavorable for wire or microcatheter crossing, which required prolonged procedural time, increased probability of complications, and reduced reimbursement. All of these factors rendered reattempt recanalization of CTO lesions considerably difficult and failed. As was demonstrated in our analysis and the study by Tanabe et al., the inability of guidewire passage through CTO lesions was the most common reason for the failure of re-PCI of CTOs [24]. The main reason of wire failure lied in lesion anatomy, as is shown in Table $2 ;>50 \%$ of lesions had an ambiguous stump, two-thirds of CTOs had an occlusion length $>20 \mathrm{~mm}, 56.1 \%$ of patients had unavailable collaterals, and $37.8 \%$ had triple vessel disease or left main plus multivessel disease. 
TAвle 3: Strategies and differences between final successful and failed cases.

\begin{tabular}{|c|c|c|c|}
\hline & Final successful reattempts $(n=172)$ & Final failed reattempts $(n=40)$ & $p$ value \\
\hline Month of intervals & $4.08 \pm 5.26$ & $3.85 \pm 5.34$ & 0.799 \\
\hline Technical success & $172(100)$ & $0(0.0)$ & - \\
\hline Procedural success & $169(98.3)$ & $0(0.0)$ & - \\
\hline CCTA preprocedure & $45(27.1)$ & $3(7.5)$ & $<0.001$ \\
\hline Dual injection & $127(73.8)$ & $17(42.5)$ & 0.003 \\
\hline \multicolumn{4}{|l|}{ Procedural approach } \\
\hline Antegrade approach & $88(51.2)$ & $15(37.5)$ & 0.119 \\
\hline Retrograde approach & $16(9.3)$ & $4(10.0)$ & 0.892 \\
\hline Hybrid approach & $68(39.5)$ & $21(52.5)$ & 0.135 \\
\hline \multicolumn{4}{|c|}{ Collaterals used in retrograde wiring } \\
\hline Septal & $69(40.1)$ & $20(50.0)$ & 0.254 \\
\hline Epicardial & $16(9.3)$ & $7(17.5)$ & 0.157 \\
\hline Bypass graft/ipsilateral & $3(1.7)$ & $0(0.0)$ & 0.402 \\
\hline Tip injection & $20(11.6)$ & $3(7.5)$ & 0.580 \\
\hline IVUS-guided wiring & $74(43.0)$ & $8(20.0)$ & 0.007 \\
\hline R-CART & $32(18.6)$ & $2(5.0)$ & 0.035 \\
\hline AGT & $26(15.1)$ & $0(0.0)$ & 0.018 \\
\hline Rotational atherectomy & $9(5.2)$ & $0(0.0)$ & 0.213 \\
\hline CrossBoss or Stingray & $11(6.4)$ & $2(5.0)$ & 0.740 \\
\hline
\end{tabular}

Values are mean \pm SD or percentage. CCTA, coronary multispiral computed tomography angiography; IVUS, intravascular ultrasound; R-CART, reversecontrolled antegrade or retrograde subintimal tracking; AGT, active greeting technique.

TABLE 4: Contrast and radiation differences.

\begin{tabular}{lcccc}
\hline & \multicolumn{2}{c}{ Final successful cases $(n=172)$} & \multicolumn{2}{c}{ Final failed cases $(n=40)$} \\
& Initial attempt & Successful reattempt & Initial attempt & Failed reattempt \\
\hline Contrast $(\mathrm{mL})$ & $249.37 \pm 142.76$ & $297.38 \pm 148.96^{\mathrm{a}}$ & $229.12 \pm 162.61$ & $317.38 \pm 184.42^{\mathrm{a}, \mathrm{b}}$ \\
Dose of X-ray (mGy) & $1739.69 \pm 1110.72$ & $2319.11 \pm 1397.31^{\mathrm{a}}$ & $1622.36 \pm 988.02$ & $2491.28 \pm 1178.14^{\mathrm{a}, \mathrm{b}}$ \\
Time of X-ray (min) & $38.63 \pm 23.59$ & $46.89 \pm 34.10^{\mathrm{a}}$ & $36.88 \pm 20.32$ & $48.82 \pm 33.66^{\mathrm{a}, \mathrm{b}}$ \\
\hline
\end{tabular}

Values are mean \pm SD. mL, milliliter; mGy, milligray. a, compared to the index attempt, $p<0.01$; b, comparison of failed reattempt vs. successful reattempt, $p<0.05$.

TABLE 5: Complications between final successful and failed reattempted cases.

\begin{tabular}{|c|c|c|c|}
\hline & $\begin{array}{l}\text { Final successful reattempts } \\
\qquad(n=172)\end{array}$ & $\begin{array}{l}\text { Final failed reattempts } \\
\qquad(n=40)\end{array}$ & $p$ value \\
\hline Procedural complications & $25(14.5)$ & $8(20.0)$ & 0.390 \\
\hline Tamponade & 0 & $3(7.5)$ & 0.004 \\
\hline Dissection/hematoma/perforation & $23(13.4)$ & $5(12.5)$ & 0.883 \\
\hline $\begin{array}{l}\text { Others (puncture site complication, wire broken, and side branch } \\
\text { occlusion) }\end{array}$ & $2(1.2)$ & 0 & 0.493 \\
\hline $\mathrm{CIN}$ & $16(9.6)$ & $2(5)$ & $<0.001$ \\
\hline MACE & 3 & 1 & 0.570 \\
\hline
\end{tabular}

Values are mean \pm SD or percentage. CIN, contrast-induced nephropathy; MACE, major adverse cardiac events (including death, myocardial infarction, and recurrent symptoms requiring immediate repeat target vessel revascularization).

Given increased complexity and risk, the retrograde approach was usually performed when antegrade crossing attempts fail or carried more risk. In the antegrade approach era, the low success rate of $65 \%-70 \%$ (even lower rate for reattempts) and high complication rate were the main barriers to CTO-PCIs [25]. The advent of the retrograde approach circumvented the limitations of the antegrade approach. Moreover, increasing knowledge and expertise could improve complex CTO-PCIs' success rate when the conventional antegrade approach was deemed unsafe or inefficient $[1,26]$. However, the choice between antegrade and retrograde approaches using wire escalation or dissection reentry methods depended on the CTO anatomy and the operator's experience, favoring timely use of a hybrid algorithm if the procedure did not progress smoothly [27]. It was very important to determine the strategy for a reattempt CTO-PCI with reference to detailed lesion information by preprocedural CCTA and dual injection during the operation. In this study, preprocedural CCTA occupied $27.1 \%$, and dual injection was employed in $73.8 \%$ of patients in final successful reattempts to provide detailed lesion characteristics, including lesion length, proximal and distal cap 


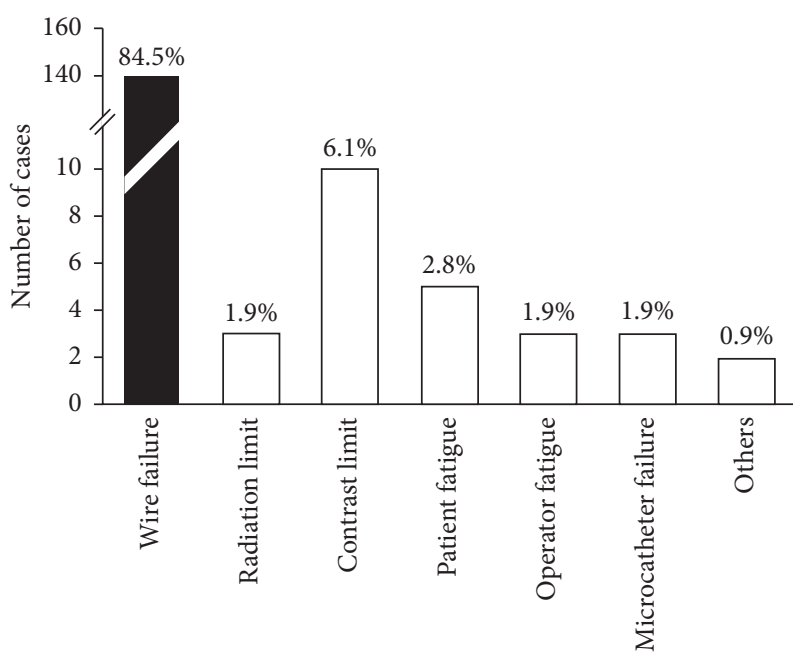

FIgURE 2: Distribution of procedural failures.

TABLE 6: Multivariable logistic regression analysis.

\begin{tabular}{lc}
\hline Parameter & $p$ value \\
\hline Sex & 0.807 \\
Age & 0.983 \\
Target lesion & 0.206 \\
J-CTO score & 0.009 \\
Month of interval & 0.076 \\
CC scale & 0.019 \\
CCTA & 0.013 \\
Dual injection & 0.002 \\
Procedural approach & 0.117 \\
IVUS-guided wiring & 0.018 \\
ADR/RDR & 0.070 \\
AGT & 0.012 \\
Parallel wiring & 0.023 \\
Rotational atherectomy & 0.229 \\
Bridging collaterals usage & 0.127 \\
In-stent occlusion & 0.342 \\
Proximal cap morphology & $<0.001$ \\
Proximal collateral & 0.018 \\
CTO length & 0.001 \\
CTO bend & 0.693 \\
Distal cap morphology & $<0.001$ \\
Distal collateral & 0.003 \\
Calcification & 0.012 \\
HTN & 0.066 \\
DM & 0.477 \\
HG & 0.820 \\
Smokers & 0.482 \\
LVEF & 0.521 \\
eGFR & 0.583 \\
Prior MI & 0.119 \\
Prior CABG & 0.084 \\
CCior PCI & 0.211 \\
\hline
\end{tabular}

CC, collateral connection; CCTA, coronary multispiral computed tomography angiography; IVUS, intravascular ultrasound; ADR, anterograde dissection and reentry; RDR, retrograde dissection and reentry; AGT, active greeting technique; HTN, hypertension; DM, diabetes mellitus; HG, hyperlipidemia; LVEF, left ventricular ejection fraction; eGFR, estimated glomerular filtration rate; $\mathrm{MI}$, myocardial infarction; CABG, coronary artery bypass grafting; PCI, percutaneous coronary intervention. morphology, and the extension and morphology of the collateral branches, which helped drive the strategy choice or switch. CTO crossing is often easier in the retrograde direction because the distal CTO cap is often less fibrocalcific, more tapered, and exposed to lower pressure than the proximal cap [28].

In addition to choosing an appropriate procedural approach and strategy, it is noteworthy that a suitable combination of techniques and devices was also of high importance. In our analysis of final successful reattempts, IVUS-guided CTO wiring to resolve proximal cap ambiguity, R-CART, AGT, and CrossBoss and Stingray systems were used at a high percentage. Particularly, retrograde wire externalization facilitated by AGT appeared feasible and safe at our center [29].

The optimal time threshold for change continued to be debated and heavily dependent on operator expertise. The operators decided the use of all available crossing strategies and prompt changes in techniques and equipment. Expertise in all CTO crossing strategies was crucial for achieving the best possible procedural outcomes, especially for complex lesions. Antegrade wire escalation was often associated with lower complication rates and was often preferred as the initial crossing strategy if technically feasible. However, for complicated CTO lesions, antegrade wiring was related to a low success rate. Retrograde and antegrade dissection and reentry remained essential for the successful recanalization of more complex CTOs. In subsequent reattempts, all operators who had performed a career minimum of 300 CTO cases mastered all the available techniques and the appropriate combination of devices (antegrade dissection reentry (ADR) and retrograde dissection reentry (RDR)) in the hybrid algorithm. Referral to a more experienced operator could be helpful, especially if the CTO is of high complexity or there was a previous failed attempt.

The overall incidences of procedure-related complications and MACE were low in this study, similar to previous reports $[24,30]$, except that the CIN rate was higher in both finally successful and failed reattempted patients. Consequently, CTO-PCI in reattempted lesions was as safe as initially attempted CTO-PCI.

It should be noted that forty CTO lesions in forty patients remained unopened despite multiple attempts, twenty-four patients were referred to $\mathrm{CABG}$, and the rest chose medical therapy with residual symptoms. These lesions were compromised with high J-CTO scores and lacked fair interventional collaterals, which means no chance for an antegrade or retrograde approach, and successful interventional revascularization might be limited even though expert operators endeavored reattempted CTO-PCIs.

4.1. Study Limitations. Although we aimed to take an allcomer population, most operators were reliant upon referrals from other cardiologists, and the treatment policy was largely determined by the individual operator. Therefore, these results would be unlikely generalizable to other lessexperienced CTO-PCI operators. 


\section{Conclusions}

Considering lesion complexity and prior failure, reattempted CTO-PCIs could still achieve an overall success rate of $81.1 \%$ by high-volume operators with acceptable complications.

\section{Data Availability}

The primary data are available on request from the author.

\section{Disclosure}

Mingqiang Fu and Shufu Chang are the co-first authors.

\section{Conflicts of Interest}

The authors declare that they have no conflicts of interest.

\section{Acknowledgments}

This work was supported by grants from the National Natural Science Foundation of China (81700225 to Mingqiang $\mathrm{Fu}$ ), the Clinical Science and Technology Innovation Project of Shanghai Shenkang Hospital Development Center (SHDC12018X05 to Jianying Ma), and Project of Shanghai Municipal Health Commission (201940152 to Shufu Chang).

\section{Supplementary Materials}

Correlation analysis is conducted to determine the related indexes with procedural outcomes. It revealed that higher J-CTO score, collaterals existed in proximal or distal lesions, lesion length $\geq 20 \mathrm{~mm}$, and severe lesion calcification were negatively correlated with procedural success. In contrast, higher CC scale, preoperation CCTA application, dual injection, IVUS-guided wiring, AGT, and tapered cap existed in both proximal and distal lesions were positively correlated with successful recanalization. (Supplementary Materials)

\section{References}

[1] J. Y. Ma, J. Y. Qian, L Ge et al., "Retrograde approach for the recanalization of coronary chronic total occlusion: collateral selection and collateral related complication," Chinese Medical Journal, vol. 126, no. 6, pp. 1086-1091, 2013.

[2] O. M. Jeroudi, M. E. Alomar, T. T. Michael et al., "Prevalence and management of coronary chronic total occlusions in a tertiary Veterans Affairs hospital," Catheterization and Cardiovascular Interventions, vol. 84, no. 4, pp. 637-643, 2014.

[3] B. E. Claessen, G. D. Dangas, G. Weisz et al., "Prognostic impact of a chronic total occlusion in a non-infarct-related artery in patients with ST-segment elevation myocardial infarction: 3-year results from the HORIZONS-AMI trial," European Heart Journal, vol. 33, no. 6, pp. 768-775, 2012.

[4] L. P. Hoebers, M. M. Vis, B. E. Claessen et al., "The impact of multivessel disease with and without a co-existing chronic total occlusion on short- and long-term mortality in ST-elevation myocardial infarction patients with and without cardiogenic shock," European Journal of Heart Failure, vol. 15, no. 4, pp. 425-432, 2013.
[5] S. Windecker, P. Kolh, F. Alfonso et al., "ESC/EACTS guidelines on myocardial revascularization: the task force on myocardial revascularization of the European society of Cardiology (ESC) and the European association for cardiothoracic surgery (EACTS)developed with the special contribution of the European association of percutaneous cardiovascular interventions (EAPCI)," European Heart Journal, vol. 35, no. 37, pp. 2541-2619, 2014.

[6] L. J. Shaw, D. S. Berman, D. J. Maron et al., "Optimal medical therapy with or without percutaneous coronary intervention to reduce ischemic burden," Circulation, vol. 117, no. 10, pp. 1283-1291, 2008.

[7] G. S. Werner, V. Martin-Yuste, D. Hildick-Smith et al., "A randomized multicentre trial to compare revascularization with optimal medical therapy for the treatment of chronic total coronary occlusions," European Heart Journal, vol. 39, no. 26, pp. 2484-2493, 2018.

[8] P. Marechal, L. Davin, O. Gach et al., "Coronary chronic total occlusion intervention: utility or futility," Expert Review of Cardiovascular Therapy, vol. 16, no. 5, pp. 361-367, 2018.

[9] A. Quadros, K. C. Belli, J. E. T. Paula et al., "Chronic total occlusion percutaneous coronary intervention in Latin America," Catheterization and Cardiovascular Interventions, vol. 96, no. 5, pp. 1046-1055, 2020.

[10] P. Tajti, K. Alaswad, D. Karmpaliotis et al., "Procedural outcomes of percutaneous coronary interventions for chronic total occlusions via the radial approach: insights from an international chronic total occlusion Registry," JACC. Cardiovascular Interventions, vol. 12, no. 4, pp. 346-358, 2019.

[11] Y. Suzuki, E. Tsuchikane, O. Katoh et al., "Outcomes of percutaneous coronary interventions for chronic total occlusion performed by highly experienced Japanese specialists," JACC: Cardiovascular Interventions, vol. 10, no. 21, pp. 2144-2154, 2017.

[12] E. L. Hannan, Y. Zhong, A. K Jacobs et al., "Patients with chronic total occlusions undergoing percutaneous coronary interventions: characteristics, success, and outcomes," Circ Cardiovasc Interv, vol. 9, no. 5, Article ID e003586, 2016.

[13] E. S. Brilakis, S. Banerjee, D. Karmpaliotis et al., "Procedural outcomes of chronic total occlusion percutaneous coronary intervention," JACC: Cardiovascular Interventions, vol. 8, no. 2, pp. 245-253, 2015.

[14] T. T. Tsai, M. A. Stanislawski, K. A. Shunk et al., "Contemporary incidence, management, and long-term outcomes of percutaneous coronary interventions for chronic coronary artery total occlusions," JACC: Cardiovascular Interventions, vol. 10, no. 9, pp. 866-875, 2017.

[15] Y. Morino, M. Abe, T. Morimoto et al., "Predicting successful guidewire crossing through chronic total occlusion of native coronary lesions within 30 minutes," JACC: Cardiovascular Interventions, vol. 4, no. 2, pp. 213-221, 2011.

[16] P. Fefer, M. L. Knudtson, A. N. Cheema et al., "Current perspectives on coronary chronic total occlusions," Journal of the American College of Cardiology, vol. 59, no. 11, pp. 991-997, 2012.

[17] M. B. McEntegart, A. A. Badar, F. A. Ahmad et al., "The collateral circulation of coronary chronic total occlusions," EuroIntervention, vol. 11, no. 14, pp. e1596-e1603, 2016.

[18] S. A. Harding, E. B. Wu, S. Lo et al., "A new algorithm for crossing chronic total occlusions from the asia pacific chronic total occlusion club," JACC: Cardiovascular Interventions, vol. 10, no. 21, pp. 2135-2143, 2017.

[19] P. Tajti, D. Karmpaliotis, K. Alaswad et al., "The hybrid approach to chronic total occlusion percutaneous coronary 
intervention," JACC: Cardiovascular Interventions, vol. 11, no. 14, pp. 1325-1335, 2018.

[20] D. V. Daniels, S. Banerjee, K. Alaswad et al., "Safety and efficacy of the hybrid approach in coronary chronic total occlusion percutaneous coronary intervention: the Hybrid Video Registry," Catheterization and Cardiovascular Interventions, vol. 91, no. 2, pp. 175-179, 2018.

[21] E. Maccariello, "Contrast induced nephropathy," J Bras Nefrol, vol. 38, no. 4, pp. 388-389, 2016.

[22] T. Ramunddal, L. P. Hoebers, J. P. Henriques et al., "Chronic total occlusions in Sweden--a report from the Swedish coronary angiography and angioplasty Registry (SCAAR)," PLoS One, vol. 9, no. 8, Article ID e103850, 2014.

[23] G. E. Christakopoulos, G. Christopoulos, M. Carlino et al., "Meta-analysis of clinical outcomes of patients who underwent percutaneous coronary interventions for chronic total occlusions," The American Journal of Cardiology, vol. 115, no. 10, pp. 1367-1375, 2015.

[24] M. Tanabe, K. Kodama, K. Asada, and T. Kunitomo, "Lesion characteristics and procedural outcomes of re-attempted percutaneous coronary interventions for chronic total occlusion," Heart and Vessels, vol. 33, no. 6, pp. 573-582, 2018.

[25] J. A. Suero, S. P. Marso, P. G. Jones et al., "Procedural outcomes and long-term survival among patients undergoing percutaneous coronary intervention of a chronic total occlusion in native coronary arteries: a 20-year experience," Journal of the American College of Cardiology, vol. 38, no. 2, pp. 409-414, 2001.

[26] G. S. Werner, M. Ferrari, S. Heinke et al., "Angiographic assessment of collateral connections in comparison with invasively determined collateral function in chronic coronary occlusions," Circulation, vol. 107, no. 15, pp. 1972-1977, 2003.

[27] L. Maiello, A. Colombo, R. Gianrossi et al., "Coronary angioplasty of chronic occlusions: factors predictive of procedural success," American Heart Journal, vol. 124, no. 3, pp. 581-584, 1992.

[28] J. Irving, "CTO pathophysiology: how does this affect management?" Current Cardiology Reviews, vol. 10, no. 2, pp. 99-107, 2014.

[29] J. Ge, L. Ge, B. Zhang et al., "Active greeting technique: a mother-and-child catheter based technique to facilitate retrograde wire externalization in recanalization of coronary chronic total occlusion," Science Bulletin, vol. 63, no. 23, pp. 1565-1569, 2018.

[30] M. F. Khan, E. S. Brilakis, C. S. Wendel, and H. Thai, "Comparison of procedural complications and in-hospital clinical outcomes between patients with successful and failed percutaneous intervention of coronary chronic total occlusions: a meta-analysis of observational studies," Catheterization and Cardiovascular Interventions, vol. 85, no. 5, pp. 781-794, 2015. 